\title{
ESTIMATIVA DOS TEORES DE CARBONO POR ESPECTROSCOPIA NO INFRAVERMELHO EM Merostachys skvortzovii (BAMBUSOIDAE)
}

\author{
Felipe Scheibe Dallagnol ${ }^{1}$, Carlos Roberto Sanquetta ${ }^{2}$, Silvana Nisgoski $^{3}$, Francelo Mognon $^{4}$ \\ ${ }^{1}$ Eng. Florestal, Doutorando em Eng. Florestal, UFPR, Curitiba, PR, Brasil - fsdallagnol@gmail.com \\ ${ }^{2}$ Eng. Florestal, Dr., Depto. de Ciências Florestais, UFPR, Curitiba, PR, Brasil - sanquetta@ufpr.br \\ ${ }^{3}$ Eng $^{\mathrm{a}}$. Florestal, Dr ${ }^{\mathrm{a}}$., Depto. de Engenharia e Tecnologia Florestal, UFPR Curitiba, PR, Brasil - silnis@ yahoo.com \\ ${ }^{4}$ Biólogo, M.Sc., Doutorando em Eng. Florestal, UFPR. Curitiba, PR, Brasil - mognonf@gmail.com
}

Recebido para publicação: 26/04/2012 - Aceito para publicação: 22/03/2013

\begin{abstract}
Resumo
Os métodos para a estimação do carbono no solo e na vegetação são críticos para as estimativas, geralmente são onerosos e envolvem muito trabalho de campo e de laboratório. Essa justificativa reforça a necessidade de uma metodologia rápida e de simples operação e resposta. Dessa forma, o objetivo deste trabalho é validar a utilização do infravermelho para gerar estimativas que possam satisfazer as premissas de rapidez e baixo custo nas estimativas com alta correlação aos teores reais de carbono encontrados na biomassa. Para que este trabalho fosse realizado, foram comparados os teores provenientes da análise de carbono pelo método da combustão e pelas estimativas provenientes da análise do infravermelho (NIR). Os espectros sofreram diferentes pré-tratamentos antes da modelagem, para obtenção dos teores de carbono. Com relação aos teores de carbono encontrados na espécie em questão, eles oscilaram entre $35,87 \%$ e $45,58 \%$, com uma média geral para os teores de $42,63 \%$. O modelo que melhor representou as variações dos teores de carbono foram os espectros que sofrerem o pré-tratamento através da aplicação da segunda derivada, resultando em um erro de validação interna de 0,822 e um coeficiente de calibração $\left(\mathrm{R}^{2}\right)$ 0,764.

Palavras-chave: Bambu; Nir; método não destrutivo.
\end{abstract}

\begin{abstract}
Carbon yeld estimation by infrared spectroscopy in Merostachys skvortzovii (Bambusoidae). The estimation methods for the soil's and vegetation's carbon are critical for the estimates, they are often expensive and involve too much field work and laboratory, reinforcing the need for a methodology of rapid and simple operation and response. So, the objective of this research is to validate the use of infrared analysis to generate estimates that can satisfy the assumptions. The concentrations of carbon from the analysis by the combustion method were compared to the estimates derived from the infrared analysis (NIR), the spectra experienced different pretreatments before modeling in order to obtain the carbon content. In relation to carbon content found in the concerned species, they ranged between $35.87 \%$ and $45.58 \%$ with an overall average for the contents of $42.63 \%$. The model that better represented variations of the carbon were the spectra under pretreatment by applying the second derivative, resulting in a validation error of 0.822 and an internal calibration coefficient ( $\left.\mathrm{R}^{2}\right)$ of 0.764 . Keywords: Bamboo; Nir; Non-destructive method.
\end{abstract}

\section{INTRODUÇÃO}

Levantamentos de biomassa e carbono tornam-se cada vez mais importantes para obtenção de um panorama local e nacional sobre a potencialidade de fixação e o comportamento da quantidade de carbono que pode estar presente na biomassa.

As florestas têm sua importância destacada, já que elas constituem o maior reservatório de carbono de todos os ecossistemas terrestres e funcionam, em muitos casos, como sumidouros de carbono, o que corrobora a sua inclusão em projetos no Mecanismo de Desenvolvimento Limpo (MDL). Entretanto, a fim de que se possa avaliar o potencial para a geração de créditos de carbono, é de fundamental importância que se proceda à quantificação do carbono estocado nas florestas (RIBEIRO, 2007). Os bambus, presentes em boa parte do planeta, constituintes do ambiente florestal e da sucessão 
ecológica, ocupam na América Latina uma área estimada em 11 milhões de hectares (SANTOS, 2008). São tipicamente considerados como gramíneas arbustivas, mas apresentam alguns representantes com comportamento arborescente. Essas espécies contribuem significativamente para a manutenção e contenção de alguns tipos de solo e para a fixação de carbono em seus tecidos vegetais. Dessa forma, a quantificação do carbono fixado na biomassa florestal é uma ferramenta geradora de dados primários de informação, que podem vir a ser usados na proposição de projetos no âmbito do MDL. Entretanto, os métodos para a estimação do carbono no solo e na vegetação são críticos para todos os métodos subsequentes, fato que reforça a necessidade de uma metodologia rápida, de baixo custo e possível de ser replicada (RAVINDRANATH; OSTWALD, 2008).

Merostachys skvortzovii Sendulski é uma espécie que domina o sub-bosque de povoamentos florestais e de florestas do meio-oeste do estado do Paraná, sendo que essa espécie possui um ciclo entre uma floração e outra de aproximadamente 30 anos. Após sua floração e produção de sementes, a taquara seca e torna-se um substrato altamente inflamável, que em caso de combustão é capaz de gerar incêndios horizontais e verticais de grandes proporções (SANTOS, 2008). É caracterizada como sendo uma espécie de bambu de colmos lenhosos, com rizomas do tipo paquimorfo, caracterizando o tipo de desenvolvimento simpodial. Seus colmos são retos, com leve curvatura no ápice, e atingem altura máxima entre 4 e $6 \mathrm{~m}$. Os nós são salientes e apresentam leve pubescência esbranquiçada (SCHWARZBACH, 2008).

O objetivo deste trabalho é realizar a análise de carbono dos compartimentos da biomassa da espécie de bambu Merostachys skvortzovii pelo método de combustão e avaliar as correlações existentes entre os teores encontrados pela análise de dados proveniente do infravermelho, identificando qual o melhor método para obter estimativas confiáveis dos teores de carbono.

\section{METODOLOGIA}

As amostras analisadas de Merostachys skvortzovii proveem de propriedades pertencentes às Indústrias Pedro N. Pizzatto, localizadas no município de General Carneiro, no estado do Paraná, possuindo latitude $26^{\circ} 25^{\prime} 39^{\prime \prime}$ sul e longitude $51^{\circ} 18^{\prime} 56^{\prime \prime}$ oeste, sendo o município mais meridional do Paraná, estando a uma altitude de aproximadamente 980 metros. O município de General Carneiro está localizado na região mais fria do Paraná. Durante o inverno registram-se temperaturas abaixo de zero, com geadas frequentes.

Os procedimentos metodológicos iniciaram com a identificação em campo dos indivíduos da espécie. As formações nativas dessa espécie foram localizadas e identificadas e constituíam-se em remanescentes do último ciclo da espécie, ou seja, formações que ainda não haviam secado com o processo do fim do ciclo, entre 2006 e 2008. Após a localização das formações nativas da espécie, utilizaram-se 20 (vinte) exemplares, que foram selecionados com diferentes diâmetros na base do colmo. A amplitude diamétrica da base do colmo variou entre 9,3 e $14 \mathrm{~cm}$, com uma média de $12,15 \mathrm{~cm}$. A amplitude das circunferências à altura do peito (CAP) variou entre 9,0 e 13,5 cm, com uma média de $11,45 \mathrm{~cm}$, e a altura total dos bambus variou entre 7,1 e 10,8 metros, com média em 8,59 metros. Esses exemplares foram coletados em cinco diferentes áreas definidas em campo.

A caracterização morfológica dos exemplares da espécie foi baseada em critérios apresentados em estudos similares, como os de Torezan e Silveira (2000), Taylor e Zischeng (1987), Embaye et al. (2005), Veblen et al. (1980), os quais descrevem características morfológicas de algumas espécies de bambu, porém esses estudos oferecem apenas um indicativo da maturidade dos exemplares, não garantindo informações sobre a idade e nem mesmo sobre a própria maturidade dos exemplares. Não obstante, essas descrições morfológicas foram utilizadas para separação em compartimentos e sua identificação, o que colaborou significativamente para a distinção e compreensão do arranjo da biomassa em espécies de bambu. O processo de coleta da biomassa de bambu para a análise de carbono baseou-se no método proposto por Sanquetta e Balbinot (2004), adaptada para as espécies de bambu. Essa metodologia propõe o particionamento do objeto de estudo em compartimentos que sejam passíveis de identificação, no caso, rizoma, folhas, galhos e colmo, e esse particionamento também pode ser realizado em porções relativas, como no caso em que foi realizado com o compartimento colmo, subdividido em porção inferior e superior.

Após a coleta de biomassa em campo, subamostras foram preparadas para serem levadas ao laboratório para secagem, moagem e análise de carbono por via seca (combustão), como método de referência, para o uso de dados do infravermelho próximo (NIR - Near infrared). Em síntese, o processamento em laboratório foi efetuado de tal modo que, no final do processo, fossem obtidas amostras secas e moídas, na quantidade de aproximadamente 10 gramas, para as análises dos teores de 
carbono e para as avaliações no equipamento de infravermelho. No total, foram obtidas 100 amostras, sendo 20 repetições em cada compartimento.

$\mathrm{Na}$ análise no NIR, cada espectro representa um vetor de dados (1 X 1556), referente à quantidade de informações que estão entre as bandas de absorção selecionadas. Foram coletados 300 espectros na faixa do infravermelho, que se encontra entre $4.000 \mathrm{~cm}^{-1}$ e $10.000 \mathrm{~cm}^{-1}$, sendo que de cada amostra foram feitos três espectros, a fim de se captar a variação existente em toda a amostra. No momento das análises, houve uma redução na quantidade de espectros, sendo que os dados de cada amostra geraram um espectro médio. Para a avaliação, os espectros do infravermelho foram submetidos a dois processos de análise multivariada, a análise de componentes principais (PCA) e a regressão por mínimos quadrados parciais (MQP) (MORGANO; MORIYA, 2002; CONZEN, 2006; CARNEIRO, 2008; SAMISTRARO, 2008).

Os dados foram ajustados para obtenção dos teores de carbono pelo método de regressão multivariada, intitulada quadrados parciais (PLS). Os dados provindos do equipamento de infravermelho passaram por pré-tratamentos, para ressaltar as características dos espectros, tendo sido verificados e comparados diferentes pré-tratamentos. Os métodos empregados foram a correção de multiplicativa de sinal (MSC) (NAES et al., 2002), a aplicação da segunda derivada Noris-Gap (HONORATO et al., 2007) e a combinação desses dois métodos.

A avaliação dos modelos de calibração multivariada é feita para calcular o erro dos modelos de calibração, sendo empregado o RMSE (Root Mean Square Error), conforme equação 1, em que $n$ é o número de espectros, $y_{i}$ e $\hat{y}_{i}$ são os valores determinados pelo método padrão do equipamento LECO e o valor predito pelo modelo do PLS, respectivamente, utilizando as amostras do conjunto de calibração para validação cruzada (RMSECV) ou de predição externa (RMSEP).

$$
R M S E=\sqrt{\frac{\sum_{i=1}^{n}(\mathrm{yi}-\hat{\mathrm{y}})^{2}}{n}}
$$

Após ser selecionado o melhor pré-tratamento, são retiradas do grupo de calibração amostras outliers, que são determinadas em função do erro RMSE de calibração ou distância Mahalanobis que apresentam, de modo a diminuir o erro RMSE de calibração ou o número de fatores PLS utilizados. A distância Mahalanobis refere-se à distância entre cada espectro e um espectro médio determinado pelo software, e quando essa distância é superior à unidade, a amostra referente a esse espectro é considerada outlier. Uma análise qualitativa dos espectros também ajuda na determinação de amostras outliers, uma vez que o agrupamento de amostras permite a identificação de amostras que são diferentes das restantes, pelas mais variadas razões. Por fim, o conjunto de amostras foi dividido em diferentes espécies, correspondentes às amostras usadas na calibração e ao grupo das amostras usadas na validação. Esse passo torna o modelo mais robusto, capaz de responder a alterações do sistema.

\section{RESULTADOS}

$\mathrm{Na}$ tabela 1 estão os resultados obtidos na análise do teor de carbono para os cinco compartimentos da espécie Merostachys skvortzovii. O teor médio, considerando todos os cinco compartimentos, é de $42,63 \%$, os valores mais contrastantes foram observados no compartimento rizoma.

Tabela 1. Variação dos teores de carbono nos compartimentos.

Table 1. Change in carbon stocks in the compartments.

\begin{tabular}{lccccc}
\hline Variável & $\begin{array}{c}\text { Colmo } \\
\text { inferior }(\boldsymbol{\%})\end{array}$ & $\begin{array}{c}\text { Colmo } \\
\text { superior (\%) }\end{array}$ & Folhas (\%) & $\begin{array}{c}\text { Galhos } \\
\text { vivos (\%) }\end{array}$ & Rizoma (\%) \\
\hline Limite inferior & 39,077 & 40,752 & 36,544 & 40,126 & 35,870 \\
Média & 43,045 & 43,379 & 40,874 & 42,091 & 42,420 \\
Limite superior & 44,533 & 44,721 & 43,274 & 43,489 & 45,578 \\
Coeficiente de variação & $3,84 \%$ & $2,00 \%$ & $4,76 \%$ & $1,76 \%$ & $17,51 \%$ \\
\hline
\end{tabular}

Na figura 1a é possível observar as variações das médias dos teores de carbono em cada compartimento da biomassa, sendo que as maiores variações foram observadas no compartimento rizoma. Essas variações podem ser explicadas pelas dificuldades de delimitação da área e pela incerteza das idades de cada rizoma. A figura $1 \mathrm{~b}$ mostra o resultado da análise de variância e do teste de Tukey para apontar as diferenças entre as médias de cada compartimento, apontando que existem diferenças significativas entre os teores de carbono encontrados para o colmo e as folhas e entre as folhas e o rizoma. 


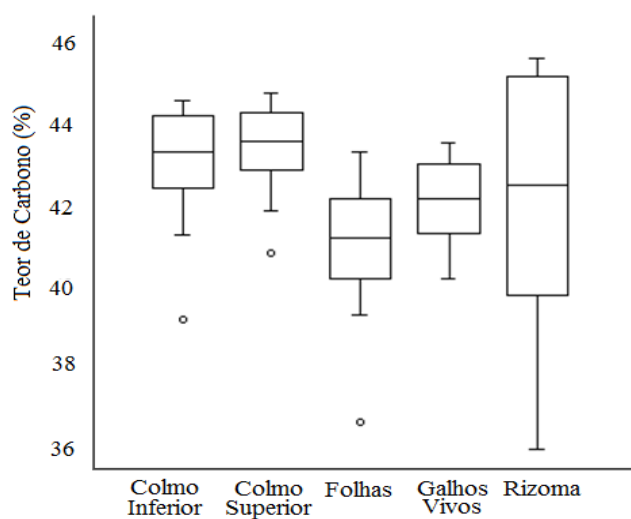

Colmo
Inferior

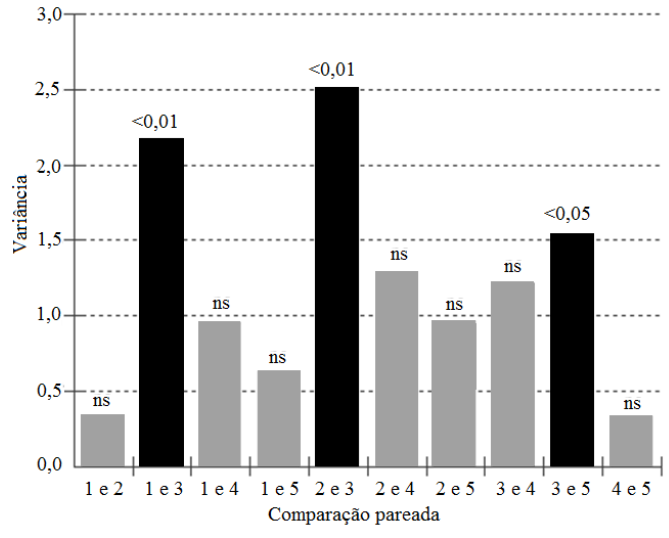

(b)

Figura 1. (a) - Variação dos teores de carbono em cada compartimento da biomassa; (b) - Resultado da análise de variância entre os teores de cada compartimento. $(<0,01$ : diferença significativa ao nível de 99\% de probabilidade; <0,05: diferença significativa ao nível de 95\% de probabilidade; ns: diferença não significativa).

Figure 1. (a) - Variation of carbon content in each compartment of biomass; (b) - Result of analysis of variance between the contents of each compartment. $(<0.01$ : significant difference at $99 \%$ probability; <0.05: significant difference at $95 \%$ probability; ns: nonsignificant difference).

No conjunto global das amostras estudadas existem grupos de amostras que se sabe de início e pela análise de variância que possuem diferenças significativas entre si. Uma das principais diferenças reside no fato de os teores de carbono terem sidos analisados por compartimentos e assumirem assim diferentes valores. Outra diferença está na cor das amostras, que provocam alterações em nível espectral, fazendo com que esse grupo de amostras fique diferenciado dos restantes. Conhecendo as características das amostras, foi realizada uma análise qualitativa somente com os espectros NIR obtidos, ou seja, uma análise de componentes principais que permitisse concluir se haveriam e quais seriam os agrupamentos existentes, podendo-se dessa forma prosseguir para a construção de modelos PLS com cada grupo de amostras obtido, de modo a construir modelos mais precisos para a previsão de resultados. $\mathrm{O}$ resultado dessa análise pode ser observado na figura 2.



Figura 2. Diagrama dos compartimentos e sua distinção no infravermelho para a espécie Merostachys skvortzovii. MF: folhas; MGV: galhos; MCS: colmo superior; MCI: colmo inferior; MR: rizoma.

Figure 2. Diagram of the Biomass compartments and their distinction in the infrared for Merostachys skvortzovii. MF: leaves; MGV: branches; MCS: top culm; MCI: lower culm; MR: rhizome. 
A análise de todos os espectros coletados na região do infravermelho próximo para a espécie Merostachys skvotzovii resultaram em diferentes modelos. Na tabela 2 encontram-se os valores para os melhores quatro ajustes dos dados, levando em conta a quantidade de variáveis latentes, o erro de calibração (RMSEC) e de validação (RMSEP), juntamente com os coeficientes de correlação de erro e calibração (MORGANO; MORIYA, 2002; NISGOSKI, 2005; SAMISTRARO, 2008; CARNEIRO, 2008).

Tabela 2. Resultado dos melhores ajustes para os teores de carbono para Merostachys skvortzovii.

Table 2. Results for the best models for carbon yeld of Merostachy__skvortzovii.

\begin{tabular}{|c|c|c|c|c|c|}
\hline Tipo de pré-tratamento & VAR. Latentes & RMSEC & RMSEP & $\mathbf{R}^{2}$ Cal. & $\mathbf{R}^{2}$ Val. \\
\hline SEM PRÉ-TRATAMENTO 4 & 6 & 0,7682 & 0,8901 & 0,6567 & 0,5537 \\
\hline SEM PRÉ-TRATAMENTO 3 & 7 & 1,1198 & 1,343 & 0,6249 & 0,4754 \\
\hline SEM PRÉ-TRATAMENTO 2 & 8 & 1,1115 & 1,3608 & 0,6304 & 0,4614 \\
\hline SEM PRÉ-TRATAMENTO 1 & 13 & 0,8269 & 1,3914 & 0,7955 & 0,4369 \\
\hline $\mathrm{MSC}+2^{\circ}$ Derivada 4 & 5 & 0,7397 & 0,9129 & 0,7022 & 0,5603 \\
\hline $\mathrm{MSC}+2^{\circ}$ Derivada 3 & 4 & 0,8788 & 1,0796 & 0,7644 & 0,6541 \\
\hline $\mathrm{MSC}+2^{\circ}$ Derivada 2 & 7 & 0,6212 & 0,8991 & 0,8823 & 0,76 \\
\hline $\mathrm{MSC}+2^{\circ}$ Derivada 1 & 7 & 0,5018 & 1,3542 & 0,9231 & 0,4557 \\
\hline MSC 4 & 7 & 0,7255 & 0,8658 & 0,7250 & 0,6220 \\
\hline MSC 3 & 8 & 0,6758 & 0,8691 & 0,7614 & 0,6191 \\
\hline MSC 2 & 8 & 1,0826 & 1,338 & 0,6426 & 0,4687 \\
\hline MSC 1 & 11 & 0,8391 & 1,3465 & 0,7852 & 0,4619 \\
\hline $2^{\circ}$ Derivada $4 *$ & 9 & 0,6603 & 0,8220 & 0,7654 & 0,6478 \\
\hline $2^{\circ}$ Derivada 3 & 5 & 0,8571 & 1,0722 & 0,7759 & 0,6588 \\
\hline $2^{\circ}$ Derivada 2 & 8 & 0,5555 & 0,8609 & 0,9059 & 0,7800 \\
\hline $2^{\circ}$ Derivada 1 & 7 & 0,5448 & 1,3745 & 0,9094 & 0,4393 \\
\hline
\end{tabular}

*Melhor correlação.

O modelo que apresentou os melhores resultados foi o modelo tratado com o método da segunda derivada, baseado no método de Noris-Gap, sendo que o erro de validação interna foi de 0,822 e o coeficiente de correlação de calibração foi de 0,7654 . Nas Figuras 3 e 4 é possível observar as correlações entre o modelo de calibração e da validação interna, respectivamente. Esse modelo foi escolhido entre os outros porque apresentou os pares de valores tanto do RMSEC quanto do RMSEP baixos, comparados aos outros, e um conjunto de $\mathrm{R}^{2} \mathrm{Cal}$. e $\mathrm{R}^{2} \mathrm{~V}$ al. altos.

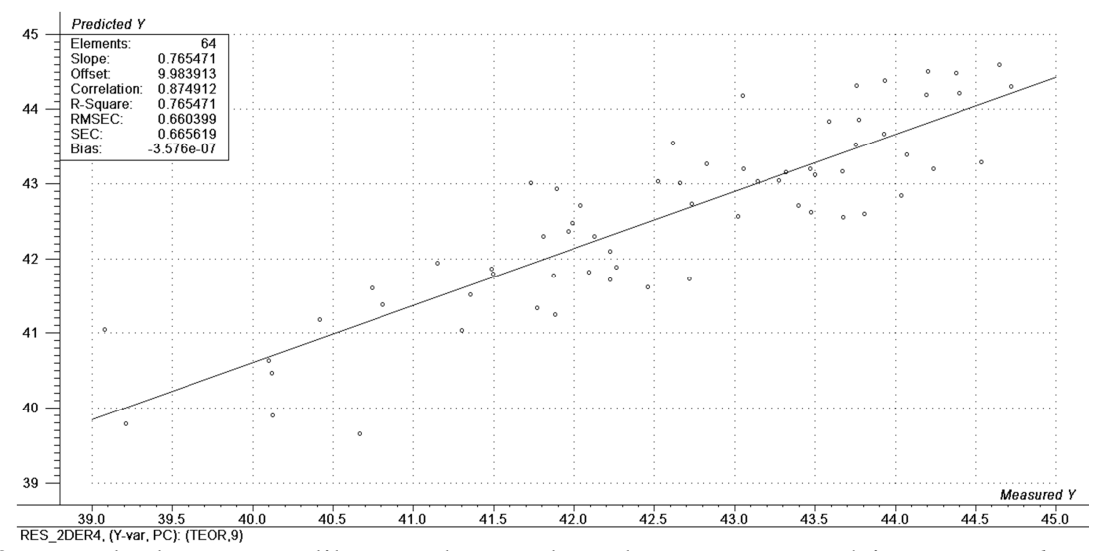

Figura 3. Resultado para a calibração do teor de carbono para a espécie Merostachys skvortzovii.

Figure 3. Results for the best calibration carbon yeld model for Merostachys skvortzovii.

É válido destacar que a espécie Merostachys skvortzovii apresentou uma amostra presente no conjunto de validação externa que continha a presença de umidade e fungo que se desenvolveu após a análise de carbono, motivo pelo qual ela foi removida do conjunto de calibração externa. $\mathrm{O}$ fato de essa 
amostra estar presente elevou consideravelmente o erro atribuído à validação externa, o que comprometia o melhor modelo com um erro de 3,2973 pontos percentuais, para uma média geral de teores de carbono da espécie Merostachys skvortzovii de 42,3617, que, em termos percentuais, significa 7,78\% de erro na predição dos teores de carbono. Por esse motivo, o conjunto de validação externa contém apenas 24 elementos.

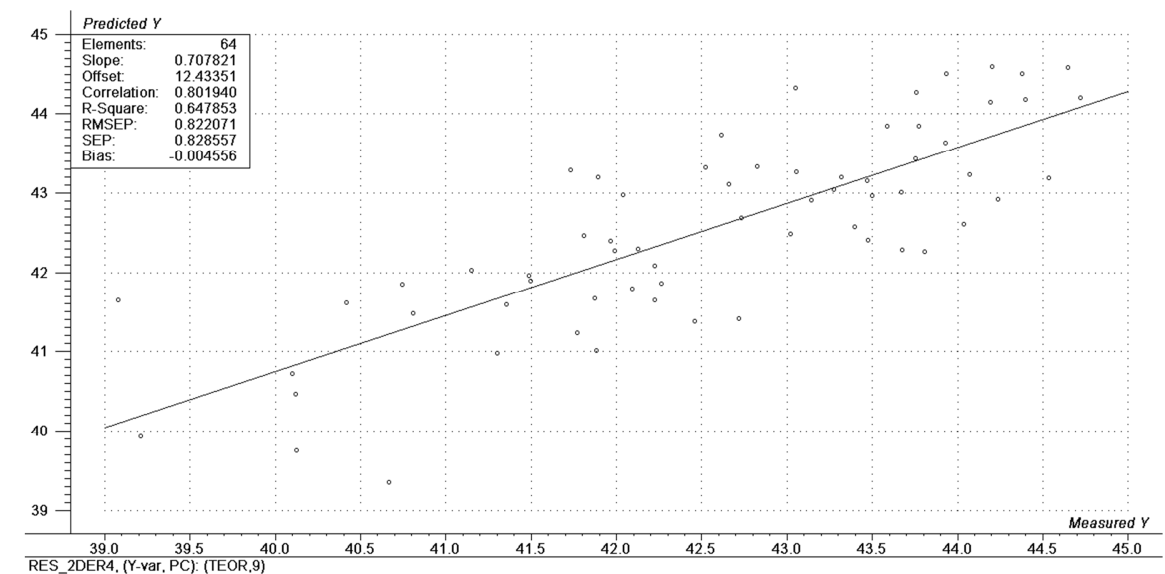

Figura 4. Resultado para a validação interna do teor de carbono para a espécie Merostachys skvortzovii.

Figure 4. Results for the internal validation to the best calibration carbon yeld model for Merostachys skvortzovii.

Na figura 5 é possível observar a correlação entre os valores de referência e os obtidos através da análise NIR, para o modelo que melhor se ajustou aos dados reais.



Figura 5. Predição dos teores de carbono para a espécie Merostachys skvortzovii.

Figure 5. Prediction of carbon contents for the species Merostachys skvortzovii.

Considerando as variações e o alto nível de correlação entre as variáveis, os modelos podem ser considerados significantes, quando comparados a valores encontrados na literatura para espécies de bambu que foram avaliadas pelo método do infravermelho. Um exemplo é o trabalho realizado por Jiang et al. (2007), os quais realizaram um estudo sobre a determinação do teor de holocelulose de bambu (Phyllestachys heterocycla (Carr.) Mitford cv. pubescens), por espectroscopia de infravermelho próximo por reflectância, ajustando para 54 amostras. Após as transformações feitas através da segunda derivada, foi obtido um coeficiente de correlação de 0,95 e um erro de validação de 0,76\%. Gy et al. (2007a) determinaram o conteúdo de lignina klason utilizando 54 amostras, sem tratamento prévio dos espectros, 
e obtiveram um coeficiente de correlação de calibração e de validação de 0,99 e 0,97 , respectivamente, com um erro de calibração e validação de $0,36 \%$ e $0,59 \%$, respectivamente. Em outro trabalho Gy et al. (2007b) avaliaram o modelo para predizer a holocelulose e a lignina em um espécie de bambu usando o método de regressão por quadrados parciais. O coeficiente de correlação do modelo de predição para teor de holocelulose e teor de lignina foi de 0,92 e 0,93 , respectivamente. $\mathrm{O}$ erro padrão de previsão para teor de holocelulose e teor de lignina foi de $1,04 \%$ e $0,913 \%$, respectivamente. Os resultados da previsão foram semelhantes aos dos modelos de previsão desenvolvidos pelos métodos tradicionais.

\section{CONCLUSÕES}

- Avaliando os resultados obtidos, pode-se concluir que foi possível ajustar modelos que obtivessem uma boa correlação com os dados reais de carbono e os espectros coletados na região do infravermelho analisados através da calibração por método multivariado.

- A ferramenta NIR apontou ser um caminho de rápida obtenção de dados com uma ótima correlação e baixos erros associados à predição. A possibilidade de realização da coleta de dados com baixo custo da análise contribui para um aumento do seu uso, mas ainda é necessário a calibração de amostras para que a predição dos valores seja condizente com a realidade, fato que reforça a importância da uniformidade das amostras e da sua representatividade, contemplando as características durante todo o momento das análises e na identificação de amostras anômalas. O principal exemplo foi a identificação de amostras com fungos e as com teores de umidade diferentes das outras amostras, pois obtiveram um contraste bem marcante quando comparadas com a variação normal de cada espectro de cada amostra. Por esse fato, a uniformização da amostra com relação ao tamanho das partículas, procedência e teor de umidade são fatores primordiais para obtenção de resultados satisfatórios. Dessa forma, essas características explicam significativamente o crescimento e uso do equipamento e das metodologias para avaliação de dados.

- A aplicação dos pré-tratamentos aos dados foi de extrema eficácia, pois diminuíram as interferências causadas pela luz e ressaltaram as diferenças, contribuindo para a obtenção de melhores ajustes. Em próximos estudos, considerar trabalhar com diferentes teores de umidade e amostras coletadas diretamente em campo, para a calibração de modelos externos.

\section{REFERÊNCIAS}

CARNEIRO, M. E. Classificação de lâminas de madeira de Pinus spp. por espectroscopia óptica. 106 p. Dissertação (Mestrado em Engenharia Florestal) - Setor de Ciências Agrárias, Universidade Federal do Paraná. Curitiba, 2008

CONZEN, J. P. Multivariate Calibration. A Practical Guide for the Method Development in the Analytical Chemistry. Bruker Optick GmbH. 2nd edition. 2006.

EMBAYE, K.; WEIH, M.; LEDIN, S.; CHRISTERSSON, L. Biomass and nutrient distribution in a highland bamboo forest in southwest Ethiopia: implications for management. Forest Ecology and Management, n. 204, p. 159 - 169, 2005.

GY, L.; HUANG, A. M.; QIN, T. F. Rapid modeling method for spectroscopic analysis of chemical components of bamboo. 2007 PubMed - indexed for MEDLINE. Disponível em: <http://www.ncbi.nlm.nih.gov/pubmed/19798960>. Acesso em: 20/10/2011.

GY, L.; HUANG, A. M.; WANG, G.; QIN, D. C.; JIANG, Z. H. Rapid determination of Klason lignin content in bamboo by NIR. 2007 PubMed - indexed for MEDLINE. Disponível em: <http://www.ncbi.nlm.nih.gov/pubmed/18306776>. Acesso em: 20/10/2011.

HONORATO, F. A.; BARROS NETO, B.; NASCIMENTO, M. M.; HARROP, G. R. K.; PIMENTEL, M. F Transferência de calibração em métodos multivariados, Quim. Nova, v. 30, n. 5, p. 1301 - 1312, 2007. 
JIANG, Z.; LI, G.; WANG, G.; HUANG, A. Study on determination of holocellulose content of bamboo by near-infrared reflectance spectroscopy, 2007. Disponível em: <http://en.cnki.com.cn/Article_en/CJFDTOTAL-LCHX200701003.htm〉. Acesso em: 20/10/2011.

MORGANO, M. A.; MORIYA, C.; FERREIRA, M. M. C. Determinação quantitativa do teor de sacarose em açúcar cristal por espectroscopia FT-IR/ATR e Regressão Multivariada. Brazilian Journal of Food Technology, 2002.

NAES, T.; ISAKSSON, T.; FEARN, T.; DAVIES, T. A user-friendly guide to multivariate calibration and classification. NIR Publications. 2002.

NISGOSKI, S. Espectroscopia no infravermelho próximo no estudo de características da madeira e papel de Pinus taeda L. 160 f. Tese (Doutorado em Engenharia Florestal) - Setor de Ciências Agrárias, Universidade Federal do Paraná, Curitiba, 2005.

RAVINDRANATH, N. H.; OSTWALD, M. Carbon inventory methods: handbook for greenhouse gas inventory, carbon mitigation and roundwood production projects. Ravindranath N. H., Ostwald M. - xix, 304 p. - Advances in global change research; 2. - New York: Springer, 2008.

RIBEIRO, S. C. Quantificação do estoque de biomassa e análise econômica da implementação de projetos visando a geração de créditos de carbono em pastagem, capoeira e floresta primária. Programa de Pós-Graduação em Ciência Florestal Universidade Federal de Viçosa, julho de 2007.

SAMISTRARO, G. Propriedades químicas e físicas da polpa e papel kraft por espectroscopia no infravermelho próximo (NIR). $106 \mathrm{f}$. Dissertação (Mestrado em Engenharia Florestal) - Setor de Ciências Agrárias, Universidade Federal do Paraná. Curitiba, 2008.

SANQUETTA, C. R.; BALBINOT, R. Metodologias para determinação de biomassa florestal. In: SANQUETTA C. R. et al. Fixação de carbono: atualidades, projetos e pesquisas. Curitiba: editado pelos autores, p. 77 - 93, 2004.

SANTOS, K. Impacto do controle mecânico de taquaras (BAMBUSOIDEAE) sobre a regeneração de uma área de Floresta Ombrófila Mista. 127 f. Dissertação (Mestrado em Engenharia Florestal) Universidade Federal do Paraná. Curitiba, 2008.

SCHWARZBACH, L. L. C. Bambus e taquaras: avaliação e perspectivas de uso sustentável. 60 p. Dissertação (Mestrado em Engenharia Florestal) - Pós-Graduação em Engenharia Florestal, Universidade Federal do Paraná. Curitiba, 2008.

TAYLOR, A. H.; ZISHENG, Q. Culm dynamics and dry matter production of bamboos in the Wolong and the Tangjiahe Giant Panda Reserves, Sichuan, China. Journal of Applied Ecology, n. 2, p. 419 433, 1987.

TOREZAN, J. M. D.; SILVEIRA, M. The biomass of bamboo (Guadua weberbaueri Pilger) in open forest of the southwestern Amazon. Ecotropica, n. 6, p. 71 - 76, 2000.

VEBLEN, T. T.; SCHLEGEL, F. M.; ESCOBAR, B. R. Dry matter production of two species of bamboo (Chusquea culeou and C. tenuiflora) in South-Central Chile. Journal of Ecology, n. 68, p. 397 - 404, 1980. 